\title{
Ocorrência de Giardia sp. e Cryptosporidium sp. em Leopardus weidii de vida livre
}

\author{
Occurrence of Giardia sp. and Cryptosporidium sp. in Leopardus weidii of wild life
}

\author{
Camila Belmonte Oliveira' ${ }^{\mathrm{I}}$ João Fábio Soares ${ }^{\mathrm{I}}$ Aleksandro Schafer da Silva ${ }^{\mathrm{I}}$ \\ Marcos Kipper da Silva ${ }^{\mathrm{I}}$ Edson Luis SalomãoII Silvia Gonzalez Monteiro ${ }^{\mathrm{III}}$
}

\begin{abstract}
Leopardus weiidi (gato-maracajá) é um mamífero RESUMO neotropical de ampla distribuição no Brasil. No município de Cachoeira do Sul, RS, um exemplar foi capturado para tratamento, quando se coletou uma amostra de fezes, que foi analisada pelo método de centrífugo-flutuação com sulfato de zinco para pesquisa de parasitos. Foi observada elevada infecção por cistos de Giardia sp. e oocistos de Cryptosporidium sp. . Com base nos resultados, conclui-se que L. weeidi é hospedeiro desses protozoários.

Palavras-chave: Leopardus weiidi, Giardia sp., Cryptosporidium sp.

\section{ABSTRACT}

Leopardus weiidi (gato-maracajá) is a neotropical mammal with has a wide distribution in Brazil. In the municipality of Cachoeira do Sul, RS, a fecal sample from a specimen captured for treatment was collected and analyzed by the centrifugal-flotation method with zinc sulfate for research of parasites. A large number of cysts of Giardia sp. and oocysts of Cryptosporidium sp. was observed. The results indicate that L. weeidi is a host of these protozoa.
\end{abstract}

Key words: Leopardus weiidi, Giardia sp, Cryptosporidium sp.

O espécime de felino selvagem, Leopardus wiedii, conhecido popularmente como gato-maracajá, apresenta hábito noturno, terrestre, arbóreo e solitário. Alimenta-se de pequenos mamíferos, aves e répteis, sendo encontrado em matas de todo o território nacional, exceto na Região Nordeste (OLIVEIRA \& CASSARO, 1999). Alguns estudos evidenciam o gato doméstico como hospedeiro de protozoários zoonóticos dos gêneros Giardia e Cryptosporidium, podendo ser parasitado por $\boldsymbol{C}$. parvum, $\boldsymbol{C}$. muris e $\boldsymbol{C}$. felis, sendo que a transmissão zoonótica depende da espécie do protozoário e da suscetibilidade do hospedeiro. O parasitismo por Cryptosporidium sp. pode ser subclínico ou apresentar manifestações clínicas como diarréia, sendo os filhotes mais suscetíveis, provavelmente devido à imaturidade do seu sistema imune (HUBER et al., 2002).

A Giardia sp. é um protozoário intestinal amplamente distribuído pelo mundo (MACHADO et al., 2001), que causa doença clínica moderada, severa ou pode ocorrer sem sinais clínicos. Durante seu ciclo evolutivo, há dois estágios de vida: a forma cística e a forma trofozoíta (BECK et al., 2005), sendo o cisto a forma infectante (MACHADO et al., 2001). Em animais jovens, causa diarréia intermitente com comprometimento da digestão e absorção de alimentos, acarretando desidratação e perda de peso, que podem levar à morte (MUNDIM et al., 2003).

ICurso de Medicina Veterinária, Universidade Federal de Santa Maria (UFSM), Santa Maria, RS, Brasil.

IIMédico Veterinário autônomo.

IIIDepartamento de Microbiologia e Parasitologia, Centro de Ciência da Saúde (CCS), UFSM. Faixa de Camobi, Km 9, 97105-900, Prédio 20, Sala 4232, Santa Maria, RS, Brasil. Autor para correspondência. 
Este trabalho tem como objetivo relatar o parasitismo natural por Giardia sp. e Cryptosporidium sp. em gato-maracajá (L. weidii), no município de Cachoeira do Sul, Rio Grande do Sul.

O animal foi capturado em uma propriedade rural, no interior do município, devido a apresentar-se apático, fraco e com manchas de sangue no pêlo, onde foram observadas escoriações profundas. O felino foi conduzido ao zoológico da cidade, sendo anestesiado para realização de exame clínico, tratamento das lesões e coleta de amostras. A amostra fecal foi coletada diretamente da ampola retal e processada para detecção de ovos, cistos e oocistos de parasitos pela técnica de centrífugo-flutuação com sulfato de zinco (HOFFMANN, 1987).

Na amostra analisada, observou-se elevado parasitismo por cistos de Giardia sp. e oocistos de Cryptosporidium sp.. No entanto, o animal não apresentava sinais clínicos aparentes dessas enfermidades, tais como diarréia e vômito. Ovos de Toxocara sp. também foram verificados na amostra. Em estudo realizado com gatos domésticos, HILL et al. (2000) observaram a ocorrência de organismos entéricos zoonóticos em animais que apresentavam diarréia no Estado do Colorado, EUA, sendo encontrados nas análises fecais Cryptosporidium parvum e Giardia sp., dois gêneros de protozoários identificados neste estudo em gato-maracajá de vida livre. Em trabalhos realizados no Brasil por GENNARI et al. (1999) e RAGOZO et al. (2002), nos quais a ocorrência de parasitos gastrintestinais em gatos domésticos foi avaliada, os protozoários Giardia sp. e Cryptosporidium parvum também foram encontrados em amostras diarréicas e não-diarréicas.

Até o momento, existem poucos registros no país de levantamentos parasitológicos sobre a infecção de felinos selvagens por Giardia sp. e Cryptosporidium sp.. MÜLLER et al. (2005), em estudos realizados com felinos (Panthera tigris, Panthera leo, Felis serval, Panthera onca, Puma concolor e Leopardus tigrinus) do Zoológico de Pomerode e do Parque Ecológico Zoobotânico de Brusque, ambos do Estado de Santa Catarina, observaram infecção por Giardia sp. em 38,5\% dos animais, sendo que 23,1\% apresentavam infecção mista com helmintos. No Parque Ecológico Zoobotânico de Brusque, $40 \%$ dos exemplares de Felis serval pesquisados apresentaram infecção por Giardia sp.; entretanto, nas amostras provenientes de $\mathbf{L}$. weidii, Giardia sp. e Cryptosporidium sp., não foram observados esses parasitas, sendo esta a primeira descrição desses protozoários nesta espécie de felídeos.
PATTON \& RABINOWITZ (1994) relataram, em estudo realizado com 92 amostras fecais coletadas de várias espécies de felídeos selvagens da Tailândia, 94\% de amostras positivas para cistos de Giardia sp., confirmando a ampla distribuição desse protozoário entre os felídeos. Com base nos resultados, conclui-se que o gato-maracajá é hospedeiro em potencial de protozoários como Giardia sp. e Cryptosporidium sp., assim como os felinos em geral, havendo possibilidade de disseminar esses parasitos no meio, infectando outras espécies de mamíferos.

\section{REFERÊNCIAS}

BECK, C. et al. Freqüência da infecção por Giardia lamblia (Kunstler, 1882) em cães (Canis familiaris) avaliada pelo Método de Faust e cols. (1939) e pela Coloração da Auramina, no município de Canoas, RS, Brasil. Ciência Rural, v.35, n.1, p.126-130, 2005.

GENNARI, S.M. et al. Ocorrência de protozoários e helmintos em amostras de fezes de cães e gatos da cidade de São Paulo. Brazilian Journal of Veterinary Research and Animal Science, v.36, n.2, p.87-91, 1999.

HILL, S.L. et al. Prevalence of enteric zoonotic organisms in cats. Journal American Veterinary Medical Association, v.216, n.5, p.687-692, 2000.

HOFFMAN, R.P. Diagnóstico de parasitismo veterinário. Porto Alegre: Sulina, 1987. 156p.

HUBER, F. et al. Comparação entre infecção por Cryptosporidium sp. e por Giardia sp. em gatos sob dois sistemas de criação. Revista Brasileira de Parasitologia Veterinária, v.11, n.1, p.7-12, 2002.

MACHADO, R.L.D. et al. Evaluation of four techniques for diagnosis of Giardia lamblia in children's stool from Belém city, Para State, Brazil. Revista da Sociedade Brasileira Medicina Tropical, v.34, n.1, p.91-93, 2001.

MULLER, G.C.K. et al. Freqüência de parasitas intestinais em felinos mantidos em zoológicos. Arquivos Brasileiros de Medicina Veterinária e Zootecnia, v.57, n.4, p.559-561, 2005.

MUNDIM, S.J.S. et al. Freqüência de Giardia spp. por duas técnicas de diagnóstico em fezes de cães. Arquivos Brasileiros de Medicina Veterinária e Zootecnia, v.55, n.6, p.770773, 2003.

OLIVEIRA, T.G.; CASSARO, K. Guia de identificação dos felinos brasileiros. São Paulo: Sociedade dos Zoológicos do Brasil, 1999. 60p

PATTON, S.; RABINOWITZ, A.R. Parasites of wild felidae in Thailand: a coprological survey. Journal of Wildlife Disease, v.30, n.3, p.472-475, 1994.

RAGOZO, A.M.A. et al. Ocorrência de parasitos gastrointestinais em fezes de gatos das cidades de São Paulo e Guarulhos. Brazilian Journal of Veterinary Research and Animal Science, v.39, n.5, p.244-246, 2002. 